Proyecciones

Vol. 17, $\mathrm{N}^{\circ}$ 2, pp. 167-176, December 1998

Universidad Católica del Norte

Antofagasta - Chile

\title{
ON THE PARABOLIC DIRICHLET TO NEUMANN FUNCTIONAL
}

\author{
CLAUDIO CUEVAS \\ Universidad de la Frontera, Chile
}

\begin{abstract}
We prove the injectivity of the linearization of the Parabolic Dirichlet to Neumann Functional in a bounded domain of $\mathbf{R}^{n}$ under suitable transversality condition.
\end{abstract}




\section{Introduction and Statement of the Result}

Let $\bar{\Omega}$ be a compact manifold of dimension $n$ with smooth boundary $\partial \Omega$ and $\operatorname{Met}(\bar{\Omega})$ denote the set of all Riemannian metrics $g$ on $\bar{\Omega}$. We consider the mixed problem for the heat equation:

$$
\begin{aligned}
& L_{g} u:=\frac{\partial u}{\partial t}-\Delta_{g} u=0, \quad \text { in } \Omega \times(0, T), \\
& u=f \text { on } \Gamma=\partial \Omega \times(0, T), f \in C_{0}^{\infty}(\Gamma), \\
& u(x, 0)=0 \text { in } \Omega .
\end{aligned}
$$

There is a unique solution to (1.1); hence we may define the parabolic Dirichlet to Neumann map as the linear operator $\Lambda_{g}: C_{0}^{\infty}(\Gamma) \rightarrow C^{\infty}(\Gamma)$ given by

$$
\Lambda_{g} f=\left.d u \cdot \nu_{g}\right|_{\Gamma}=\left.\frac{\partial u}{\partial \nu_{g}}\right|_{\Gamma},
$$

where $u$ is the unique solution to (1.1) and $\nu_{g}$ is the $g$-interior unit normal to $\partial \Omega$. The parabolic Dirichlet to Neumann Functional:

$$
\begin{aligned}
\Lambda: \operatorname{Met}(\bar{\Omega}) & \longrightarrow O_{p}(\Gamma), \\
g & \longmapsto \Lambda_{g},
\end{aligned}
$$

where $O_{p}(\Gamma)$ denotes the space of all linear operators from $C_{0}^{\infty}(\Gamma)$ into $C^{\infty}(\Gamma)$, is known to be invariantly defined on the orbit obtained by the action over $\operatorname{Met}(\bar{\Omega})$ of the group $\mathcal{D}$ of all diffeomorphisms $\psi$ of $\bar{\Omega}$, each of which restricts to the identity on $\partial \Omega$. In fact, any such $\psi$ can be used to construct a new metric, the pull-back metric, $\psi^{*} g$, such that $\Lambda_{\psi^{*} g}=\Lambda_{g}$. A natural conjecture is that this is the only obstruction to the injectivity of $\Lambda$. This article may be considered as a first step towards this claim.

The elliptic Dirichlet to Neumann map $\left.h \longmapsto \frac{\partial v}{\partial \nu_{g}}\right|_{\partial \Omega}$, where $v$ solves the Dirichlet problem $\Delta_{g} v=0$ in $\Omega,\left.v\right|_{\partial \Omega}=h$, was treated by several authors and is closely related to the physical problem referred as Electrical Impedance Tomography, of determining the conductivity of a body from measurement of voltage potencials and corresponding current fluxes at the boundary (see Uhlmann, 1992). The hyperbolic Dirichlet to Neumann map was considered in the articles (Cardoso \& Mendoza, 1996), (Lee, Mendoza, Sylvester \& Uhlmann) and (Uhlmann, 1992).

For fixed $g$, we consider the following map:

$$
\mathcal{D} \ni \psi \stackrel{A_{g}}{\longmapsto} \psi^{*} g \in \operatorname{Met}(\bar{\Omega}) .
$$


It is easy to see that the tangent space $T_{I} \mathcal{D}$ of $\mathcal{D}$ at the identity mapping $I$ is the vector space $\Gamma_{0}(T \bar{\Omega})$ of all smooth vector fields on $\bar{\Omega}$ which vanish on $\partial \Omega$. On the other hand, the tangent space $T_{g} \operatorname{Met}(\bar{\Omega})$ of $\operatorname{Met}(\bar{\Omega})$ at $g$ is the vector space $\Gamma\left(S^{2} \bar{\Omega}\right)$ of all smooth sections of symmetric 2-tensor on $\bar{\Omega}$. We introduce respectively on $\Gamma_{0}(T \bar{\Omega})$ and on $\Gamma\left(S^{2} \bar{\Omega}\right)$, the inner products

$$
\begin{gathered}
\langle X, Y\rangle=\int_{\bar{\Omega}} g(X, Y) v_{g}, \quad X, Y \in \Gamma_{0}(T \bar{\Omega}), \\
\langle\langle m, l\rangle\rangle=\frac{1}{n} \int_{\bar{\Omega}} \operatorname{tr}(\hat{m} \circ \hat{l}) v_{g}, \quad m, l \in \Gamma\left(S^{2} \bar{\Omega}\right),
\end{gathered}
$$

where $v_{g}$ (resp., $t r$ ) denotes the volume element (resp., the trace) associated to $g$ and $\hat{m}$ is the unique linear map (in fact a section of $\operatorname{End}(T \bar{\Omega})$ ) defined by

$$
g(\hat{m} u, v)=m(u, v), \quad \text { for all } \quad u, v \in \Gamma(T \bar{\Omega}) .
$$

Of course, $\hat{g}$ is the identity on $\Gamma(T \bar{\Omega})$ and the factor $1 / n$ in (1.6) is taken so as to have $\langle\langle g, g\rangle\rangle=\operatorname{vol}_{g}(\bar{\Omega})$. By definition, the formal linearization $A_{g}^{\prime}:=A_{g}^{\prime}[I]$, of $A_{g}$ at $I$ is the linear map $A_{g}^{\prime}: \Gamma_{0}(T \bar{\Omega}) \longrightarrow \Gamma\left(S^{2} \bar{\Omega}\right)$ given by

$$
A_{g}^{\prime}(X)=\left.\frac{d}{d s} A_{g}\left(X^{s}\right)\right|_{s=0},
$$

where $X^{s}$ is the flow associated to the vector fields $X$ (i.e. a mapping $(-\varepsilon, \varepsilon) \ni s \longmapsto X^{s} \in \mathcal{D}, \varepsilon>0$, such that, $X^{0}=I$ and $\left.\left.\frac{d}{d s} X^{s}\right|_{s=0}=X\right)$.

Similarly we consider, the formal linearization of $\Lambda$ at $g$, as the linear $\operatorname{map} \Lambda_{g}^{\prime}: \Gamma\left(S^{2} \bar{\Omega}\right) \rightarrow O_{p}(\Gamma)$, defined by

$$
\Lambda_{g}^{\prime}(m)=\left.\frac{d}{d s}\left(\Lambda_{g_{s}}\right)\right|_{s=0},
$$

where $g_{s}$ is a smooth curve on $\operatorname{Met}(\bar{\Omega}), s \in(-\varepsilon, \varepsilon)$, such that, $g_{0}=g$ and $\left.\frac{d}{d s} g_{s}\right|_{s=0}=m$.

Let $\left(A_{g}^{\prime}\right)^{*}$ denote the formal adjoint of $A_{g}^{\prime}$ with respect to the inner products (1.5) and (1.6). We state the following:

Conjecture 1.1. Let $m \in \Gamma\left(S^{2} \bar{\Omega}\right)$ and assume that (a) $\Lambda_{g}^{\prime}(m)=0$, (b) $\left(A_{g}^{\prime}\right)^{*}(m)=0$. Then $m$ is identically zero. 
Remark 1.1. The Condition (b) in Conjecture 1.1 is obviously necessary. In fact, the range of $A_{g}^{\prime}$ is contained in the kernel of $\Lambda_{g}^{\prime}$. Therefore, we should expect $\Lambda_{g}^{\prime}$ be injective on a "Transversal" subspace of the range of $A_{g}^{\prime}$; hence we shall refer to Condition (b) as the Transversality Condition.

The main result of this paper is:

Theorem 1.1. Conjecture 1.1 holds if $\bar{\Omega}$ is a bounded domain of $\mathbf{R}^{n}, n \geq$ 2 , with smooth boundary $\partial \Omega, g$ is the euclidean metric e and $m \in \Gamma_{0}\left(S^{2} \bar{\Omega}\right)$ is a symmetric 2-tensor on $\mathbf{R}^{n}$ which is supported on $\bar{\Omega}$.

The article is organized as follows: In Section 2 we prove some estimates relative to the heat equation. In Section 3 we exhibit invariant formulas for $A_{g}^{\prime},\left(A_{g}^{\prime}\right)^{*}$ and $\Lambda_{g}^{\prime}$. In Section 4 we present the proof of Thcorem 1.1. It is worth while mentioning that in the unidimensional case, i.e. $\bar{\Omega}=[0,1]$, the unit interval, we obtain that $\Lambda_{g}=\Lambda_{h}$ if and only if there exists a diffeomorphim $\psi:[0,1] \longrightarrow[0,1]$, which preserves orientation and consequently, fixes the boundary, such that $\psi^{*} g=h$.

\section{Estimates}

Let $\Omega$ be a bounded domain of $\mathbf{R}^{n}$, with smooth boundary $\partial \Omega$ and let $P(x, D)$ be a second order differential operator of the form

$$
P(x, D)=-\sum_{i, j} \frac{\partial}{\partial x_{i}}\left(a^{i j} \frac{\partial}{\partial x_{j}}\right)+\sum_{j} b^{j} \frac{\partial}{\partial x_{j}}+c,
$$

with coefficients in $C^{\infty}(\bar{\Omega})$. We assume that $\left(a^{i j}\right)$ is real and positive definite in $\bar{\Omega}$ and that $P$ is symmetric with respect to a density $\gamma d x$ with $0<\gamma \in C^{\infty}(\bar{\Omega})$ :

$$
(P(x, D) u, v)_{\gamma}=(u, P(x, D) v)_{\gamma}, \quad u, v \in C^{\infty}(\bar{\Omega}),
$$

where

$$
(u, v)_{\gamma}=\int u \bar{v} \gamma d x
$$

(The density simplifies coordinates changes but should be disregarded as otherwise irrelevant). Let $\mathcal{P}$ be the operator defined by $P(x, D)$ in $L_{\gamma}^{2}(\Omega)$ with Dirichlet boundary conditions. We have $u \in \mathcal{D}_{\mathcal{P}}$ if and only if 
$P(x, D) u=f \in L^{2}$ and $u$ is in the closure $\stackrel{\circ}{H}$ of $C_{0}^{\infty}(\bar{\Omega})$ in $H_{1}(\bar{\Omega})$. Then we have (see Section 17.5 of Hörmander, 1985),

$$
\begin{gathered}
\|u\|_{(1)}^{2} \leq C_{1}(\mathcal{P} u, u), \quad u \in \mathcal{D}_{\mathcal{P}}, \\
\|u\|_{(k+2)} \leq C_{k}\|\mathcal{P} u\|_{(k)}, \quad u \in \mathcal{D}_{\mathcal{P}},
\end{gathered}
$$

where we have used the notation

$$
\|u\|_{(k)}^{2}=\sum_{|\alpha| \leq k}\left\|D^{\alpha x} u\right\|^{2} .
$$

We shall also write $H_{(k)}(\bar{\Omega})$ for the set of all $u$ with $D^{\alpha} u \in L^{2}(\Omega),|\alpha| \leq k$, with the norm just defined.

We shall denote by $P_{z}(x, D)$ an elliptic operator of the same form as before, except that it now depends on a parameter $z \in K$, where $K$ is a compact convex body in $\mathbf{R}^{N}, N \geq 1$. We assume that the coefficients of $P_{z}$ are in $C^{\infty}(\bar{\Omega} \times K)$ and that $(2.4)$ is valid uniformly for all $P_{z}$. We have the following:

Proposition 2.1. Let $v \in C^{\infty}([0, T] \times \bar{\Omega} \times K)$ be a solution of the mixed problem

$$
\begin{aligned}
& \left(P_{z}+\frac{\partial}{\partial t}\right) v_{z}=h_{z} \text { in }[0, T] \times \bar{\Omega} \times K \\
& {[0.5 \mathrm{~cm}] v_{z}=0 \text { in }[0, T] \times \partial \Omega \times K} \\
& {[0.5 \mathrm{~cm}] v_{z}(0, x)=0 \quad \text { in } \Omega}
\end{aligned}
$$

Assume that $\partial^{j} h_{z} / \partial t^{j}=0$ when $t=0$, if $j \leq k$. Then it follows that (with the supromum taken over $[0, T] \times \bar{\Omega} \times K$ )

$$
\sum_{|\alpha|+\frac{1}{2}(n+2)<k} \sup \left|D_{t, x, z}^{\alpha} v_{z}(t, x)\right| \leq C \sum_{|\alpha| \leq k} \sup \left|D_{t, x, z}^{\alpha} h_{z}(t, x)\right| .
$$

To prove Proposition 2.1, we shall use the following:

Lemma 2.1. Let $v \in C^{\infty}([0, T] \times \bar{\Omega})$ be a solution of the mixed problem

$$
\begin{aligned}
& \left(P+\frac{\partial}{\partial t}\right) v=h \quad \text { in }[0, T] \times \bar{\Omega}, \\
& v=0 \quad \text { in }[0, T] \times \partial \Omega, \\
& v(0, x)=0 \quad \text { in } \quad \Omega .
\end{aligned}
$$


Assume that

$$
\left.\frac{\partial^{j} h}{\partial t^{j}}\right|_{t=0}=0, \quad \text { for all } j \leq k .
$$

Then it follows that

$$
\begin{gathered}
\sum_{j=0}^{k}\left\|D_{t}^{k-j} v(t, \bullet)\right\|_{(j)} \\
\leq C_{k}\left(\int_{0}^{t}\left\|D_{s}^{k} h(s, \bullet)\right\| d s+\sum_{j=0}^{k-1}\left\|D_{t}^{k-1-j} h(t, \bullet)\right\|_{(j)}\right) .
\end{gathered}
$$

Proof: Let $k=0$; since $P$ is symmetric, we have

$$
\frac{\partial}{\partial t}\|v\|_{\gamma}^{2} \leq 2 \operatorname{Re}(h, v)_{\gamma}
$$

Integrating from 0 to $t$ and letting $M^{2}=\sup _{0 \leq s \leq t}\|v(s, \bullet)\|^{2}$, we obtain

$$
\|v(t, \bullet)\|_{\gamma}^{2} \leq 2 M \int_{0}^{t}\|h(s, \bullet)\|_{\gamma} d s
$$

The same estimate holds for smaller values of $t$, so

$$
M^{2} \leq 2 M \int_{0}^{t}\|h(s, \bullet)\|_{\gamma} d s
$$

which proves (2.10) when $k=0$. If $k>0$, taking into account (2.9), assume that (2.10) is proved for $k$; we can therefore apply (2.10) to $\frac{\partial v}{\partial t}$ and $\frac{\partial h}{\partial t}$. We get (2.10) with the term for $j=k+1$ missing in the left-hand side. Now it follows from (2.5) that

$$
\|v(t, \bullet)\|_{(k+1)} \leq\|v(t, \bullet)\|_{(k+2)} \leq C_{k}\left(\|h(t, \bullet)\|_{(k)}+\left\|\frac{\partial v}{\partial t}(t, \bullet)\right\|_{(k)}\right) .
$$

This concludes the proof of Lemma 2.1.

Proof of Proposition 2.1. Let the sum in the right-hand side of (2.7) be equal to 1 . Then it follows from (2.10) that we have an uniform bound for $\left\|D_{t}^{k-j} v_{z}(t, \bullet)\right\|_{(j)}$, if $j \leq k$. Differentiating the differential equation $\left(\frac{\partial}{\partial t}+P_{z}\right) v_{z}=h_{z}$ with respect to $z_{\nu}$, we obtain

$$
\left(\frac{\partial}{\partial t}+P_{z}\right) \frac{\partial v_{z}}{\partial z_{\nu}}=\frac{\partial h_{z}}{\partial z_{\nu}}-\left(\frac{\partial P_{z}}{\partial z_{\nu}}\right) v_{z}:=H
$$

We have a bound for $\left\|D_{t}^{k-2-j} H(t, \bullet)\right\|_{(j)}$, if $j \leq k-2$, and then we may conclude, using (2.10) again, that there is an uniform bound for 
$\left\|D_{t}^{k-2-j} \frac{\partial v_{z}}{\partial z_{\nu}}(t, \bullet)\right\|_{(j)}$ if $j \leq k-2$. Continuing in this way we obtain uniform bounds for $\left\|D_{t}^{k-2|\beta|-j} \quad \partial_{z}^{\beta} v_{z}(t, \bullet)\right\|_{(j)}$ when $j \leq k-2|\beta|,|\beta| \leq \frac{k}{2}$, and the proposition is therefore a consequence of Lemma 17.5.2 in (Hörmander, 1985). The proof is complete.

\section{Invariant Formulas}

Let $\Omega$ be a bounded domain of $\mathbf{R}^{n}$ with smooth boundary $\partial \Omega$ and $g \in$ $\operatorname{Met}\left(\mathbf{R}^{n}\right)$.

(Cardoso \& Mendoza, 1996), proved that if $X \in \Gamma_{0}(T \bar{\Omega})$ and $m \in$ $\Gamma\left(S^{2} \bar{\Omega}\right)$, then it follows that

$$
\begin{gathered}
A_{g}^{\prime}(X)(\bullet, \bullet)=g\left(\nabla_{\bullet}^{g} X, \bullet\right)+g\left(\bullet, \nabla_{\bullet}^{g} X\right), \\
\left(A_{g}^{\prime}\right)^{*}(m)(\bullet)=-\frac{2}{n} \sum_{k=1}^{n} \nabla_{\mathbf{e}_{k}} m\left(\bullet, \mathbf{e}_{k}\right) .
\end{gathered}
$$

In (3.1) $\nabla^{g}$ denotes the $g$ Levi-Civita connection on $\Gamma_{0}(T, \bar{\Omega})$ and in (3.2) $\nabla$ is the $g$ Levi-Civita connection on $\Gamma\left(S^{2} \bar{\Omega}\right),\left(e_{i}\right)_{i=1, \ldots, n}$ in $\Gamma(T \bar{\Omega})$ is a $g$ orthonormal frame. We also observe that the right-hand side of $(3.2)$ is independent of the chosen orthonormal frame.

We denote by $\tilde{m} \in \tilde{\Gamma}\left(S^{2} \bar{\Omega}\right)$ the symmetric 2-tensor on $\Gamma\left(T^{*} \bar{\Omega}\right)$ corresponding to $m$ via $g$, i.e.

$$
\tilde{m}\left(U^{\#}, V^{\#}\right)=m(U, V) \text { for all } U, V \in \Gamma(T \bar{\Omega}),
$$

where $U^{\#}(\bullet)=g(U, \bullet)$.

We shall consider the "adjoint" problem

$$
\begin{gathered}
M_{g} v:=\frac{\partial v}{\partial t}+\Delta_{g} v=0 \quad \text { in } \Omega \times(0, T) \\
\left.v\right|_{\Gamma}=h \in C_{0}^{\infty}(\Gamma), \quad v(x, T)=0 \quad \text { in } \Omega .
\end{gathered}
$$

Let $g_{s},|s|<\varepsilon$, be any curve in $\operatorname{Met}(\bar{\Omega})$ such that $g_{0}=g$ and $\frac{d g_{s}}{d s}=m$. We need the following:

Proposition 3.1. The linearization of $\Lambda$ at $g \in \operatorname{Met}(\bar{\Omega})$, satisfies

$$
\left\langle\Lambda_{g}^{\prime}(m) f, h\right\rangle=\int_{0}^{T} \int_{\Omega}\left[\tilde{m}(d u, d v)+\frac{1}{2} \operatorname{tr} \tilde{m}\left(\tilde{g}(d u, d v)+v \frac{\partial u}{\partial t}\right)\right] v_{g} d t
$$

for all $f, h \in C_{0}^{\infty}(\Gamma)$, where $u$ (respect. $v$ ) is a solution of (1.1) (respect. 3.3 ), and tr $\tilde{m}$ means the trace of $\tilde{m}$ associated to $g$. 
Proof: By applying Stoke's theorem, we obtain

$$
\begin{aligned}
& \left\langle\Lambda^{\prime} g(m) f, h\right\rangle=\left.\frac{d}{d s}\right|_{s=0} \int_{0}^{T} \int_{\partial \Omega} h \frac{\partial u_{s}}{\partial \nu} v_{g_{s}}^{*} d t= \\
& =\left.\frac{d}{d s}\right|_{s=0} \int_{0}^{T} \int_{\Omega}\left[v_{s} \Delta_{g_{s}} u_{s}+\tilde{g}_{s}\left(d u_{s}, d v_{s}\right)\right] v_{g_{s}} d t,
\end{aligned}
$$

where $u_{s}$ (respect. $v_{s}$ ) is the solution of (1.1) (respect. (3.3)) with the metric $g$ replaced by $g_{s}$ and $v_{g_{s}}^{*}$ is the element of volume on $\partial \Omega$, induced by $g_{s}$.

From Proposition 2.1, we obtain that $u_{s}, v_{s}$ depend differentiably of $s$. Hence, we can write

$$
\begin{aligned}
& u_{s}=u+s \omega_{1}+O\left(s^{2}\right), \\
& v_{s}=v+s \omega_{2}+O\left(s^{2}\right),
\end{aligned}
$$

where of course $\left.\omega_{i}\right|_{\Gamma}=\left.\omega_{1}\right|_{t=0}=\left.\omega_{2}\right|_{t=T}=0, i=1,2$. If we substitute (3.6) into (3.5) and make use of the facts that $\left.\frac{d}{d s} \tilde{g}_{s}\right|_{s=0}=\tilde{m}$ and $\left.\frac{d}{d s} v_{g_{s}}\right|_{s=0}=$ $\frac{1}{2} \operatorname{tr}(\tilde{m}) v_{g}$, we obtain (3.4), after noticing that (by Stoke's Theorem),

$$
\int_{0}^{T} \int_{\Omega}\left[\tilde{g}\left(d \omega_{1}, d v\right)+\tilde{g}\left(d u, d \omega_{2}\right)\right] v_{g} d t=\int_{0}^{T} \int_{\Omega}\left[\omega_{1} \frac{\partial v}{\partial t}-\omega_{2} \frac{\partial u}{\partial t}\right] v_{g} d t .
$$

\section{Proof of Theorem 1.1}

We shall only prove Theorem 1.1 when $n \geq 3$; the case $n=2$ requires small modifications. Let $\Gamma_{0}\left(S^{2} \bar{\Omega}\right)$ denote the vector space of all smooth sections of symmetric 2 -tensor on $\mathbf{R}^{n}$ which are supported on $\bar{\Omega}$ and $m \in \Gamma_{0}\left(S^{2} \bar{\Omega}\right)$. In the case of the euclidean metric e, taking into account (3.2), we may write the Transversality Condition as

$$
\sum_{i=1}^{n} \frac{\partial}{\partial x_{i}} m_{i j}(x)=0, \quad \text { for all } \quad x \in \Omega, \quad j=1, \ldots, n,
$$

where $m_{i j}$ are functions in $C^{\infty}(\bar{\Omega})$ given by

$$
m_{i j}=m\left(\frac{\partial}{\partial x_{i}}, \frac{\partial}{\partial x_{j}}\right),
$$


and $x_{i}, i=1, \ldots, n$ are the standard euclidean coordinates in $\mathrm{R}^{n}$. We define

$$
\bar{m}_{i j}(x)=\left\{\begin{array}{l}
m_{i j}(x) \quad \text { if } \quad x \in \Omega \\
{[0.5 \mathrm{~cm}] 0 \text { if } \quad x \in \mathbf{R}^{n} \backslash \Omega .}
\end{array}\right.
$$

If we take the Fourier transform of (4.1), we obtain

$$
\mathcal{F}(\bar{m})(k) k=0, \quad \text { for all } k \in \mathbf{R}^{n} .
$$

We consider the solutions $u_{\tau}^{i}, i=1,2$, of $L_{g} u=0$ and $M_{g} v=0$ respectively, of the form

$$
u_{\tau}^{i}(x, t)=\mathrm{e}^{\left\langle x, \rho_{i}\right\rangle}\left(\frac{1}{\tau^{2}}+R_{\tau}^{i}(x, t)\right),
$$

where

$$
\begin{aligned}
& \rho_{1}=\frac{\eta}{2}+\frac{i(\tau w+k)}{2}, \\
& {[0.5 \mathrm{~cm}] \rho_{2}=-\frac{\eta}{2}+\frac{i(-\tau w+k)}{2},}
\end{aligned}
$$

with $\eta, k, w \in \mathbf{R}^{n},|w|=1, \tau \in \mathbf{R}$ such that

$$
\begin{aligned}
& \langle\eta, k\rangle=\langle k, w\rangle=\langle\eta, w\rangle=0, \\
& {[0.3 \mathrm{~cm}]|\eta|^{2}=\tau^{2}+|k|^{2} .}
\end{aligned}
$$

This guarantees that $\left\langle\rho_{i}, \rho_{i}\right\rangle=0, i=1,2$ and that therefore, the functions $e^{\left\langle x, \rho_{i}\right\rangle}$ are harmonic. As for $R_{\tau}^{i}(x, t), i=1,2$, they satisfy

$$
\left\{\begin{array}{l}
(-1)^{i+1} \frac{\partial}{\partial t} R_{\tau}^{i}-\Delta R_{\tau}^{i}-2 \rho_{i} \cdot \nabla R_{\tau}^{i}=0 \\
{\left.[0.5 \mathrm{~cm}] R_{\tau}^{i}\right|_{\Gamma}=0,} \\
{\left.[0.5 \mathrm{~cm}] R_{\tau}^{i}\right|_{t=t_{i}}=-\frac{1}{\tau^{2}}, t_{1}=0, t_{2}=T}
\end{array}\right.
$$

We obtain from the standard estimate for the mixed parabolic problem (see Egorov \& Shubin, 1991) that there exist a constant $C>0$, such that

$$
\left\|R_{\tau}^{i}\right\|+\left\|d R_{\tau}^{i}\right\|+\left\|\frac{\partial}{\partial t} R_{\tau}^{i}\right\| \leq \frac{C}{\tau^{2}}, i=1,2
$$

where $\|\bullet\|$ denotes the norm in $L^{2}(\Omega \times(0, T))$.

If we insert $u_{\tau}^{i}$ given by (4.5), into (3.4) and let $\tau$ goes to $+\infty$, we obtain (taking into account (4.3) and (4.9)) that

$$
{ }^{t} v \mathcal{F}(\bar{m})(k) v+\frac{|v|^{2}}{8} \operatorname{tr} \mathcal{F}(\bar{m})(k)=0
$$


for all $k, v \in \mathbf{R}^{n}$, with $\langle k, v\rangle=0$.

Let us take $k=(1,0, \ldots, 0)$. From (4.4) we obtain $\mathcal{F}\left(\bar{m}_{1 \alpha}\right)(k)=0$, $\alpha=1, \ldots, n$. On the other hand, if we take $v \in k^{\perp},|v|=1$, and use (4.10), we get $\operatorname{tr} \mathcal{F}(\bar{m})(k)=0$. In fact, if $v=e_{i}, i=2, \ldots, n, e_{i}$ being the coordinator vectors, then $(4.10)$ reads $\mathcal{F}\left(\bar{m}_{i i}\right)(k)+\operatorname{tr} \mathcal{F}(\bar{m})(k) / 8=0$. If we add these equalities, reminding that $\mathcal{F}\left(\bar{m}_{11}\right)(k)=0$, we get $\operatorname{tr} \mathcal{F}(\bar{m})(k)=0$. Since $\bar{m}$ is symmetric we see by using (4.10) again that $\mathcal{F}(\bar{m})(k)=0$. Rotating coordinates, it follows from the invariant formulas in Section 3 that $\mathcal{F}(\bar{m})(k)=0$, for all $k \in \mathbf{R}^{n}$, and therefore $m=0$.

\section{References}

[1] Cardoso F. and Mendoza R., "On the hyperbolic Dirichlet to Neumann functional", Comm. in Partial Diff. Equations, 21, pp. 12351252, (1996).

[2] canción pingüinoEgorov Yu. V. and Shubin, M. A., "Partial Differential Equations", Encyclopaedia of Mathematical Sciences, 30, Springer-Verlag, (1991).

[3] Hörmander L., "The Analysis of Linear Partial Differential Operators", Vol. III, Springer-Verlag, Berlin, Heidelberg, New York, Tokyo, (1985).

[4] Lee J. M. ; Mendoza, G. ; Sylvester, J. and Uhlmann, G., "Metric deformations which preserve boundary lengths", preprint.

[5] Uhlmann G., "Inverse boundary value problems and applications", Astérisque, 207, pp. 153-211, (1992).

Received : July 1998.

\section{Claudio Cuevas}

Departamento de Matemática

Universidad de la Frontera

Casilla 54-D

Temuco

Chile 